\title{
THE EFFECTS OF PRE-EXERCISE CORINTHIAN CURRANTS SUPPLEMENTATION ON ANTIOXIDANT RESPONSES
}

\author{
Athanasios Z. Jamurtas ${ }^{1,2}$, Chariklia Deli ${ }^{1,2}$, Ioannis Fatouros ${ }^{1,2}$, Maria Selemekou ${ }^{3}$, \\ Alexandros Papoutsis ${ }^{4}$, Yiannis Koutedakis ${ }^{1,2}$, Vaios Karathanos ${ }^{4}$ \\ ${ }^{1}$ Department of Physical Education and Sport Science, University of Thessaly, \\ Greece; ${ }^{2}$ Kinesiology Department, Institute for Research and Technology Thessaly \\ (IRETETH), Center for Research and Technology - Hellas (CERTH), GREECE, \\ ${ }^{3}$ Department of Biochemistry \& Biotechnology, University of Thessaly, Greece; \\ ${ }^{4}$ Department of Nutrition \& Dietetics, Harokopio University, Athens, Greece
}

The use of nutritional supplements before and during a sporting event, especially of a prolonged nature, is very common among competitors and routinely advised by exercise professionals. Corinthian currants have a high carbohydrate content making them a potentially ideal carbohydrate source for prolonged exercise. However, their effectiveness as an ergogenic aid has never been studied. Purpose: The aim of the present study was to investigate the effectiveness of corinthian currants as an ergogenic aid during prolonged exercise. Methods: Twelve (10 males, two females) healthy individuals (Body Weight: $75.3 \pm 10.9 \mathrm{~kg}$, Height: $172.8 \pm 8.1 \mathrm{~cm}, \mathrm{VO} 2 \mathrm{max}$ : $45.4 \pm 5.9 \mathrm{ml} / \mathrm{kg} / \mathrm{min}$ ) participated. Participants were asked to perform in random order one of the following three conditions: 1. Corinthian currants, 2. Glucose, 3. Water. In each condition, participants were asked to cycle for $90 \mathrm{~min}$ at an intensity corresponding to $70-75 \%$ of their predetermined $\mathrm{VO}_{2}$ max followed by an effort to exhaustion. Blood samples were collected before exercise, at 30,60 and $90 \mathrm{~min}$ of exercise, at exhaustion and $1 \mathrm{~h}$ post exercise. Blood was analysed for total antioxidant activity (TAC) and glutathione (GSH) levels. Results: TAC was significantly $(p<0.05)$ increased due to time in all three conditions without a significant interaction being observed. Furthermore, GSH was also significantly $(p<0.05)$ decreased due to time in all three conditions without a significant interaction being observed. Conclusions: These results indicate that pre-exercise supplementation with corinthian currants does not result in significant perturbations in the antioxidant system during and following an exhaustive aerobic exercise. 